\title{
Green tea (Camellia sinensis) catechins and vascular function
}

\author{
Rosalind J. Moore, Kim G. Jackson and Anne M. Minihane* \\ Department of Food Biosciences, University of Reading, Whiteknights, Reading RG6 6AP, UK \\ (Received 27 November 2008 - Revised 17 April 2009 - Accepted 29 June 2009 - First published online 15 September 2009)
}

The health benefits of green tea (Camellia sinensis) catechins are becoming increasingly recognised. Amongst the proposed benefits are the maintenance of endothelial function and vascular homeostasis and an associated reduction in atherogenesis and CVD risk. The mounting evidence for the influential effect of green tea catechins on vascular function from epidemiological, human intervention and animal studies is subject to review together with exploration of the potential mechanistic pathways involved. Epigallocatechin-3-gallate, one of the most abundant and widely studied catechin found in green tea, will be prominent in the present review. Since there is a substantial inconsistency in the published data with regards to the impact of green tea catechins on vascular function, evaluation and interpretation of the inter- and intra-study variability is included. In conclusion, a positive effect of green tea catechins on vascular function is becoming apparent. Further studies in animal and cell models using physiological concentrations of catechins and their metabolites are warranted in order to gain some insight into the physiology and molecular basis of the observed beneficial effects.

Green tea: Catechins: Flavonoids: Epigallocatechin gallate: Endothelial function: Vascular function

Aside from water, tea is the most popular beverage in the world. Green tea (Camellia sinensis) originates from China and is primarily consumed in Asia but is becoming increasingly common in Europe, in part due to its perceived health benefits. Green tea differs from black tea by the minimal fermentation of the tea leaves during production. The tea leaves are subjected to a steam or dry heat treatment before processing, inactivating the polyphenol oxidases which oxidise and consequently condense the tea leaf polyphenols, a reaction which is promoted in black tea production to form polyphenol dimers and polymers.

Endothelial function has been increasingly recognised as a biomarker of cardiovascular health, and dysfunction of the endothelial layer is recognised as an early aetiological factor in atherogenesis. There is an accumulating body of scientific literature from both human epidemiological and intervention studies which has demonstrated the positive impact of green tea catechins on vascular function. However, there is considerable intra- and inter-study variability which is likely to be attributable to study design, population characteristics including genetics and environmental factors. Furthermore, animal and cell-culture studies have provided some insight into physiological and molecular mechanisms of action. The aim of the present paper is to review current scientific evidence for the effects of green tea and green tea catechins, specifically epigallocatechin-3-gallate (EGCG), on vascular health and function.

\section{Green tea catechins}

Green tea is rich in polyphenols, about $70 \%$ of which are catechins, and is the main dietary source of catechin gallates and gallocatechins. Table 1 gives the concentrations of the different classes of phenolics in green tea and the major flavan-3-ols, which is the collective term for the catechin group of compounds. The chemical structures of the main green tea catechins are shown in Fig. 1. Other dietary sources of catechins include red wine, grapes, apples and chocolate. EGCG is one of the most abundant green tea catechins, with a cup of green tea containing 20-200 mg EGCG. It has also been suggested to be one of the most active known compounds in green tea, mediating most of the biological effects ${ }^{(1)}$ and accounting for $32 \%$ of the antioxidant potential ${ }^{(2)}$. The remaining catechins include: (-)-epicatechin ((-)-EC), (-)-epigallocatechin (EGC) and epicatechin-3-gallate (ECG), which provide 5-7, 9-12 and 9-12\% of the antioxidant potential of green tea, respectively.

Catechins are thought to be primarily responsible for the beneficial effects of green tea. In comparison, black tea contains about $30 \%$ of the catechins found in green tea and is

\footnotetext{
Abbreviations: ACE, angiotensin-converting enzyme; ADMA, asymmetric dimethylarginine; Akt, protein kinase B; EC, epicatechin; ECG, epicatechin-3-gallate; EGC, epigallocatechin; EGCG, epigallocatechin-3-gallate; eNOS, endothelial NO synthase; FMD, flow-mediated dilatation; GTE, green tea extract; HUVEC, human umbilical vein endothelial cells; ICAM, intercellular adhesion molecule; L-NAME, $N^{\omega}$-nitro-L-arginine methyl ester; PGI 2 , prostacyclin; PI3K, phosphatidylinositol-3-kinase; UGT, UDP-glucuronosyltransferase; VCAM, vascular cell adhesion molecule.

* Corresponding author: Dr Anne M. Minihane, fax +44 118931 0080, email a.m.minihane@reading.ac.uk
} 
Table 1. Phenolics in a green tea (Camellia sinensis) infusion* (Mean values and standard deviations)

\begin{tabular}{lcc}
\hline & \multicolumn{2}{c}{ Concentration (mg/l) } \\
\cline { 2 - 3 } Compound & Mean & SD \\
\hline Total flavonols & 778 & \\
Total hydroxycinnamoyl quinic acids & 451 & \\
Total gallic acid derivatives & 128 & \\
Total flavan-3-ols & 4572 & \\
(-)-Epigallocatechin gallate & 1255 & 109 \\
(-)-Epigallocatechin & 1565 & 31 \\
(-)-Epicatechin & 738 & 29 \\
(-)-Epicatechin gallate & 361 & 21 \\
(+)-Catechin & 270 & 16 \\
(+)-Gallocatechin & 383 & 5.4 \\
\hline
\end{tabular}

* Tea preparation: $3 \mathrm{~g}$ leaves in $300 \mathrm{ml}$ boiling water for $3 \mathrm{~min}^{(100)}$.<smiles>Oc1cc(O)c2c(c1)O[C@H](c1ccc(O)c(O)c1)[C@H](O)C2</smiles><smiles>Oc1cc(O)c2c(c1)O[C@H](c1cc(O)c(O)c(O)c1)[C@H](O)C2</smiles><smiles>C[C]1Cc2c(O)cc(O)cc2O[C@@H]1c1cc(O)c(O)c(O)c1</smiles>

mainly composed of complex condensation products such as thearubigins and theaflavins.

\section{Bioavailability and metabolism of epigallocatechin-3- gallate and its metabolites}

After oral administration green tea catechins are absorbed intestinally, predominately in the jejunum and ileum, with peak absorption occurring at $1.5-2.5 \mathrm{~h}$ after consumption. EGCG and ECG are thought to be mainly transported across the intestinal epithelial cell layer via paracellular diffusion. However, bioavailability is low due to instability under digestive conditions, poor absorption, and rapid metabolism and excretion, leading to only about $5 \%$ of consumed catechins appearing in the plasma on average ${ }^{(3)}$.

(b)<smiles>Oc1cc(O)c2c(c1)O[C@H](c1ccc(O)c(O)c1)[C@H](O)C2</smiles>

(d)<smiles>O=C(O[C@H]1Cc2c(O)cc(O)cc2O[C@@H]1c1ccc(O)c(O)c1)c1cc(O)c(O)c(O)c1</smiles> 
EGCG is the only known polyphenol to be mainly in the free form $(77-90 \%)$ in the plasma ${ }^{(4)}$ whereas EGC and EC are mostly in the conjugated form (31 and $21 \%$ in the free form, respectively) ${ }^{(5)}$. The extensive metabolism in the intestine and liver leads to the formation of glucoronides, sulfides and methylated metabolites ${ }^{(5,6)}$. This biotransformation alters the chemico-physical properties of the catechins, such as the mass, charge and polarity, aiding the deactivation and rapid excretion of the biologically active compounds. Green tea catechins are methylated by the enzyme catechol-O-methyltransferase, glucuronidated by UDP-glucuronosyltransferases (UGT) such as UGT1A1, 1A8 and 1A9 and sulfadated by sulfotransferases (SULT) such as SULT1A1 and 1A3. Glucuronidation and sulfation can occur to previously methylated EGCG and vice versa, forming mixed metabolites. Green tea catechins have also been shown to undergo microbial degradation in the colon, forming compounds such as valerolactones, and phenolic and benzoic acids which may be absorbed or passed out in the faeces. In comparison with the methylated, glucuronidated and sulfadated metabolites mentioned previously, studies have shown the valerolactones to appear in the blood and urine considerably later, with a lag period of $3 \mathrm{~h}$ and a peak at $7.5-13.5 \mathrm{~h}^{(7)}$.

The major products of EGCG metabolism are $4^{\prime \prime}-O$ methyl-(-)-EGCG and $4^{\prime}, 4^{\prime \prime}$ - $O$-dimethyl-(-)-EGCG (at high or low concentrations of EGCG, respectively) ${ }^{(8)}$, $(-)-5-\left(3^{\prime}, 4^{\prime}, 5^{\prime}\right.$-trihydroxyphenyl)- $\gamma$-valerolactone and $(-)-5$ $\left(3^{\prime}, 4^{\prime}\right.$-dihydroxyphenyl)- $\gamma$-valerolactone and the respective sulfates and glucuronides. EGC is metabolised to the major products $4^{\prime}$ - $O$-methyl-EGC ${ }^{(8)}, 4^{\prime}-O$-methyl-EGC-glucuronide, $4^{\prime}$ - $O$-methyl-EGC-sulfate, 5-( $3^{\prime}, 4^{\prime}$-dihydroxyphenyl)- $\gamma$-valerolactone, 5-( $3^{\prime}, 5^{\prime}$-dihydroxyphenyl)- $\gamma$-valerolactone and $(-)$ 5 -( $3^{\prime}, 4^{\prime}, 5^{\prime}$-trihydroxyphenyl)- $\gamma$-valerolactone. EC metabolites mainly include 5-( $3^{\prime}, 4^{\prime}$-dihydroxyphenyl)-valerolactone and conjugates of $\mathrm{EC}, 3^{\prime}$ and $4^{\prime}-O$-methyl- $(-)-\mathrm{EC}^{(9)}$, whilst ECG metabolites have not been found in human urine or plasma ${ }^{(10)}$.

The biological activity of green tea catechin metabolites have been shown in most cases to be limited compared with the pure form; however, a small selection of metabolites have been shown to have similar or in some cases higher activity than the parent compound. For example, Lu et al. found EGCG-3'-glucuronide and EGCG-3"'-glucuronide to have similar radical-scavenging ability compared with free EGCG, as measured by the 1,1-diphenyl-2-picrylhydryl (DPPH) radical assay ${ }^{(11,12)}$. The major metabolites of EC and $(+)$-catechin in rats, $(-)$-epicatechin-5- $O$ - $\beta$-glucuronide and $(+)$-catechin-5-O- $\beta$-glucuronide, were also found to have comparable superoxide-scavenging abilities as assessed by electron spin resonance spectrometry ${ }^{(13)}$. A limited number of cell-culture studies have also indicated that some catechin metabolites may have a different biological activity to the parent compound. Exposure of human aortic endothelial cells to catechin metabolites extracted from the plasma of (+)-catechin-administered rats was found to inhibit monocyte adhesion when cells were pretreated with the cytokine IL-1 $\beta$. The unconjugated form, $(+)$-catechin, had no such effect ${ }^{(14)}$ Further study into this area is required to provide insight into the relative potency of the major metabolites observed in human plasma and tissues.

\section{Endothelial function and reactivity}

A 'healthy' vascular endothelium is crucial for the maintenance of vascular health. This active organ plays an essential role in the intrinsic regulation of vascular tone via the secretion of vasorelaxants, such as NO, and vasoconstrictors, such as thromboxane and endothelin-1, the production of which is regulated by various hormonal, physical and motor signals received from the surrounding vascular wall and vessel lumen. The endothelium is also involved in the inhibition of smooth muscle cell proliferation, inflammation, vascular permeability, leucocyte adhesion and platelet aggregation and maintenance of the balance between prothrombotic and profibrinolytic activity, many of which are mediated by NO. It has been proposed that the initial events leading to atherosclerosis involves the inflammatory process; monocytes adhere to the injured endothelial cells, then enter into the vascular wall where they differentiate into macrophages. Cell adhesion molecules, such as E-selectin, P-selectin, vascular cell adhesion molecule (VCAM)-1 and intercellular adhesion molecule (ICAM)-1, play an important role in initiating endothelial monocyte recruitment and adhesion. Indeed, expression of VCAM-1 has been shown to be elevated in atherosclerotic lesions together with an increase in the soluble form in the sera of atherosclerotic patients ${ }^{(15,16)}$. In addition, mice deficient in P-selectin, E-selectin or ICAM-1 have shown increased protection against atherosclerosis ${ }^{(17,18)}$.

Damage or impairment of the endothelial layer can consequently have a major impact on the regulation and functioning of the vessels. Furthermore, many studies have shown endothelial dysfunction to be highly related to atherosclerosis and $\mathrm{CVD}^{(19-22)}$ to the extent that endothelial dysfunction has been deemed to be pivotal in the initiation and progression of the aforementioned disease. Vascular reactivity, which refers to changes in the blood vessels' tone and diameter in response to a neural, endocrine or physical (passive distension) stimulus, has thus been defined as a relatively sensitive prognostic indicator of vascular health and CVD risk ${ }^{(23)}$. The classical risk factors for endothelial dysfunction such as diabetes, dyslipidaemia, smoking and hypertension appear to account for less than $20 \%$ of the variation in endothelial function $^{(24)}$, leading to the recognition of novel risk factors. Substances that can prevent damage or restore endothelial function may have important clinical applications.

Green tea catechins have been shown in the majority of epidemiological studies to be significantly inversely related to CVD and have been postulated as providing protection against endothelial dysfunction. Research into the emerging area of green tea and endothelial function will be reviewed in the following sections.

\section{Epidemiological studies of green tea and CVD risk}

A number of epidemiological studies have investigated the relationship between green tea consumption and CVD risk. For example, Imai et al. ${ }^{(25)}$ conducted a cross-sectional study among 1371 men in Japan and found increased green tea consumption to be negatively associated with total cholesterol and TAG and a decreased atherogenic index (LDL-cholesterol:HDL-cholesterol ratio). In a study of 203 Japanese patients who underwent elective coronary 
angiography, green tea was found to be an independent predictor of coronary artery disease (defined as having at least one significant coronary stenosis), with an OR of 0.84 (95\% CI $0.76,0.91)$ for one or more cups of green tea per $\mathrm{d}^{(26)}$. A protective effect of green tea was also indicated in another cross-sectional study carried out by Sasazuki et al. ${ }^{(27)}$ in which 512 coronary arteriography patients were assessed on the basis of green tea consumption. Green tea was found to be inversely associated with coronary atherosclerosis in men but not women; in a subgroup of men, excluding those undergoing treatment for diabetes mellitus, the OR associated with green tea consumption of four or more cups per d was found to be $0.4(95 \%$ CI $0.2,0.9)$. However, a similar study by Hirano et al. ${ }^{(28)}$ found no association between green tea intake and coronary artery disease in 393 Japanese patients who underwent coronary angiography. They did, nevertheless, find a significant association between green tea intake and risk of myocardial infarction, with an OR of 0.58 (95\% CI 0.34, 0.98) for one or more cups per $d$.

Prospective cohort studies strengthen this association. For example, a recent study following 40530 Japanese adults aged over 11 years found green tea to be inversely associated with CVD mortality, an association which was stronger than that for all-cause mortality ${ }^{(29)}$. The multivariate hazard ratio of CVD mortality with adjustment for socio-economic status was calculated at 0.74 (95\% CI $0.62,0.89)$ for five cups or more per $\mathrm{d}^{(29)}$. Similar results were found by Nakachi et al. ${ }^{(30)}$, with a relative risk of cardiovascular mortality of 0.72 (95\% CI $0.60,1.04)$ for those consuming over ten cups per $d$ in a Japanese study population of 8552, followed up for over 11 years. None of the observational studies conducted to date has reported vascular function as a study outcome.

\section{Chronic human intervention trials of green tea and vascular function}

There are a limited number of chronic human intervention studies in the area of green tea and vascular function. The study conducted by Widlansky et al. ${ }^{(31)}$ is currently the only study to specifically investigate the effects of isolated EGCG. In this study, forty-two clinically stable CVD patients (medical history of myocardial infarction or at least one angiographically determined coronary stenosis) were given $300 \mathrm{mg}$ EGCG and vascular function measurements were taken acutely, after $2 \mathrm{~h}$, and chronically, after $14 \mathrm{~d}$ of repeated supplementation, using flow-mediated dilatation (FMD). Vascular function was significantly increased after acute $(P=0.01)$ but not chronic $(P=0 \cdot 12)$ supplementation (measurement approximately $14 \mathrm{~h}$ after the final dose). Ryu et al. ${ }^{(32)}$ also failed to find a significant improvement in vascular function, measured using pulse wave velocity in fifty-five patients with type 2 diabetes, after chronic consumption of $9 \mathrm{~g}$ green tea $(900 \mathrm{ml}$ water) for $28 \mathrm{~d}$. Conversely, Kim et al. ${ }^{(33)}$ did find a significant improvement in FMD-assessed vascular reactivity $(P<0.001)$ after $8 \mathrm{~g}$ green tea (1 litre water) per $\mathrm{d}$ for $14 \mathrm{~d}$ using a study population of twenty smokers. Similar findings were also found in a more recent study by Tinahones et al. ${ }^{(34)}$ in which fourteen healthy women had a FMD of $12 \%$ after 5 weeks of green tea extract (GTE) (daily dose of $375 \mathrm{mg}$ catechols) compared with a $5.7 \%$ vasodilation after placebo $(P=0 \cdot 02)$. A significant reduction in oxidised
LDL $(P=0 \cdot 017)$, which has been previously shown to impair endothelium-dependent dilatation, and anti-oxidised LDL IgM antibodies $(P=0 \cdot 002)$ was also found. With regards to other atherosclerotic markers, Lee et al. ${ }^{(35)}$ conducted a study using $600 \mathrm{ml}$ green tea for $28 \mathrm{~d}$ in twenty smokers and found a significant $(P<0.001$ and $P<0.05)$ reduction in soluble P-selectin and oxidised LDL, respectively. However, no change was seen for ICAM, VCAM, total antioxidant capacity, C-reactive protein and lipid profiles.

\section{Acute human intervention trials of green tea and vascular function}

In addition to the aforementioned study by Widlansky et al. ${ }^{(31)}$, a number of other acute intervention trials have also been conducted (Table 2). Jochmann et al. ${ }^{(36)}$ found an improvement in $\mathrm{FMD}_{\max }$ (maximum dilation after hyperaemia as a percentage change from diameter before hyperaemia) from $5.4 \%$ to $10.2 \%$ between baseline and $2 \mathrm{~h}$ after consumption of $500 \mathrm{ml}$ green tea in twenty-one healthy participants; no FMD effect was found with hot water. A similar placebo-controlled study by Nagaya et al. ${ }^{(37)}$ reported a $26 \%$ increase in maximum forearm blood flow after reactive hyperaemia measured $2 \mathrm{~h}$ after $400 \mathrm{ml}$ green tea compared with baseline. A more recent study conducted by Alexopoulos et al. ${ }^{(38)}$ found that FMD-assessed endothelial function increased from $4.4 \%$ to $8.1 \%$ after $6 \mathrm{~g}$ green tea, with no significant effects shown with the caffeine or water controls. However, more intervention studies are needed to confirm these findings and to examine underlying physiological mechanisms.

\section{Animal studies examining the impact of green tea catechins and vascular function}

Animal models, in particular rodents, have proved particularly useful in investigating the relative bioefficacy of different green tea catechins and also elucidate possible molecular mechanisms underlying the physiological responses.

In vivo animal green tea supplementations studies. Similar beneficial effects of green tea catechins have been found using animal models as to those observed in human studies. Antonello et al. ${ }^{(39)}$ assigned rats to drinking water with and without GTE at $6 \mathrm{mg} / \mathrm{ml}$ together with a high or low angiotensin II dose. The response of the phenylephrinecontracted arteries to the vasodilator acetylcholine was blunted by the high-dose angiotensin II, indicating a diminished endothelial-dependent relaxation, an effect which was improved with the rodents that drank the GTE. Potenza et al. ${ }^{(40)}$ also found that chronic supplementation of $200 \mathrm{mg}$ EGCG/kg per $\mathrm{d}$ to spontaneously hypertensive rats for 3 weeks significantly improved the vascular tone of mesenteric vascular beds and reduced the systolic blood pressure. Spontaneously hypertensive rats treated with both EGCG and $N^{\omega}$-nitro-L-arginine methyl ester (L-NAME; NO synthase antagonist) did not show any effect.

The assessment of evolving and established atherosclerotic lesions in apoE-null mice given daily intraperitoneal injections of EGCG $(10 \mathrm{mg} / \mathrm{kg})$ has also been investigated by Chyu et al. ${ }^{(41)}$. After 12 and $42 \mathrm{~d}$, atherosclerotic plaque size was reduced by 55 and $73 \%$, respectively, compared with the saline-injected control mice. However, this was only apparent 
Table 2. Acute studies of green tea (Camellia sinensis) or epigallocatechin gallate (EGCG) and endothelial function in humans

\begin{tabular}{|c|c|c|c|c|c|}
\hline Author & Intervention & Time $(h)$ & Subjects & Method & Effect \\
\hline Nagaya et al. (2004) $)^{(37)}$ & Green tea, $400 \mathrm{ml}$ & 2 & Twenty smokers & FBF & $\begin{array}{c}16.9(\mathrm{SD} 4.9) \text { to } 21.3(\mathrm{SD} 5.8) \mathrm{ml} / \mathrm{min} \\
\text { per } 10 \mathrm{ml} \text { tissue }(P<0.001)\end{array}$ \\
\hline Widlansky et al. $(2007)^{(31)}$ & EGCG, $300 \mathrm{mg}$ & 2 & Forty-two CVD patients & FMD & $7.1(\operatorname{SD} 4.1)$ to $8.6(\operatorname{SD} 4.7) \%(P=0.01)$ \\
\hline Jochmann et al. $(2008)^{(36)}$ & Green tea, $500 \mathrm{ml}$ & 2 & Twenty-one healthy & FMD & $5.4(\operatorname{SD~} 2.3)$ to $10.2(\operatorname{SD~} 3.0) \%(P<0.001)$ \\
\hline Alexopoulos et al. $(2008)^{(38)}$ & Green tea, $450 \mathrm{ml}$ & $0.5,1.5,2$ & Fourteen healthy & FMD & $\begin{array}{l}4.4(\mathrm{SD} 0.8) \text { to } 8.1 \%(P<0.02) \text {; peak } \\
\text { at } 0.5 \mathrm{~h}\end{array}$ \\
\hline
\end{tabular}

FBF, forearm blood flow; FMD, flow-mediated dilatation.

in evolving plaques and was not found in established lesions. A similar study which supplied GTE at $0.8 \mathrm{mg} / \mathrm{ml}$ in the drinking water of the mice for 14 weeks also found a significant reduction $(23 \%)$ in atheromatous areas and aortic weight compared with the controls ${ }^{(42)}$.

Ex vivo green tea catechin exposure to rat aortic rings. A number of studies have utilised rat thoracic aortas with exposure to various concentrations of green tea and green tea catechins and measurement of the vascular tone response $^{(40,43-46)}$ (Table 3). For example, Potenza et al. ${ }^{(40)}$ found a dose-dependent vascular relaxation after acute exposure of mesenteric vascular beds to EGCG in the range $1-100 \mu \mathrm{M}$, a response which was nullified by L-NAME (a NO synthase agonist) or the phosphatidylinositol-3-kinase (PI3K) inhibitor, wortmannin. Another study performed by Ajay et al. ${ }^{(45)}$ found similar results using an exposure range of $100-300 \mu \mathrm{M}$, with the highest concentration of the range causing a $70.9 \%$ vasorelaxant effect against phenylephrineinduced contractions. This has also been replicated by Lim et al. ${ }^{(47)}$ with GTE at concentrations of $0.3,0.6$ and $1.2 \mathrm{mg} / \mathrm{ml}$ (about 250, 500 and $1000 \mu \mathrm{M}$ ). Conversely in this study, when EGCG at 4 and $12 \mu \mathrm{g} / \mathrm{ml}(8.7$ and $26.2 \mu \mathrm{M})$ was applied, the contractile response evoked by phenylephrine was unchanged. These differing effects were also confirmed when GTE and EGCG were administered intravenously and noradrenaline-evoked arterial blood pressure responses measured. GTE $(10 \mathrm{mg} / \mathrm{kg}$ per $30 \mathrm{~min})$ resulted in a significant decrease in pressor response whilst EGCG $(1 \mathrm{mg} / \mathrm{kg}$ per $30 \mathrm{~min}$ ) had no effect.

Surprisingly, aortic ring studies using lower concentrations of EGCG $(<10 \mu \mathrm{M})$, comparable with what may be observed in human plasma, have shown a vasoconstrictive effect ${ }^{(44)}$. It does appear that the mechanisms of action of EGCG on endothelial function in aortic ring studies is dose-dependent with the postulation that lower doses $(<25 \mu \mathrm{M})$ have an impact on the vascular endothelium ${ }^{(48)}$ whilst higher doses work in an endothelium-independent manner such as by inhibition of $\mathrm{Ca}^{2+}$ influx ${ }^{(46,49)}$ or inhibition of contractile proteins, mechanisms which may not necessarily occur at concentrations ordinarily consumed and absorbed. This apparent anomaly between human and animal in vivo studies and aortic ring responses in these lower doses is not clearly understood and yet to be fully investigated.

\section{Mechanisms underlying the impact of green tea catechins on vascular function}

\section{Cell signalling}

The central role of NO in the regulation and maintenance of vascular function has led to this molecule being the focus of attention in cell-culture mechanistic studies. For example,

Table 3. Animal studies of vascular effect of green tea (GT; Camellia sinensis), green tea extract (GTE) or epigallocatechin gallate (EGCG) exposure

\begin{tabular}{|c|c|c|c|}
\hline Author & Intervention & Method used & Effect \\
\hline Sanae et al. (2002) ${ }^{(44)}$ & $10 \mu \mathrm{M}$-catechin derivatives & Ex vivo aortic ring response & $\begin{array}{l}\text { Potentiation of endothelium-dependent contraction and } \\
\text { inhibition of acetylcholine relaxation }(P<0.05)\end{array}$ \\
\hline Ajay et al. $(2003)^{(45)}$ & $0.1-0.3 \mathrm{~mm}-\mathrm{EGCG}$ & Ex vivo aortic ring response & $\begin{array}{l}\text { EGCG maximum concentration } 70.91 \text { (SD 6.1) \% } \\
\text { phenylephrine- and } 14.26 \text { (SD 5.2) \% } \mathrm{K}^{+} \text {-induced } \\
\text { contracted relaxation }\end{array}$ \\
\hline Lorenz et al. (2004) ${ }^{(48)}$ & $1-50 \mu \mathrm{M}-\mathrm{EGCG}$ & Ex vivo aortic ring response & $\begin{array}{l}\text { Dose-dependent vasodilation, significance }(P<0.05) \\
\text { reached at } 10 \mu \mathrm{M}\end{array}$ \\
\hline Alvarez et al. $(2006)^{(50)}$ & $1 \mu \mathrm{M}-1 \mathrm{~mm}-\mathrm{EGCG}$ & Ex vivo aortic ring response & $\begin{array}{l}1-30 \mu \mathrm{M} \text { potentiated induced contractions by } 30 \% \\
30 \mu \mathrm{M}-1 \mathrm{~mm} \text { biphasic constriction followed by } \\
\text { relaxation }(P<0.01)\end{array}$ \\
\hline Fitzpatrick et al. (1995) ${ }^{(46)}$ & $\mathrm{GT}, 10 \mu \mathrm{l} / \mathrm{ml}$ & Ex vivo aortic ring response & 91 (SD 0.7) \% phenylephrine-contracted vasorelaxation \\
\hline Antonello et al. $(2007)^{(39)}$ & GTE, $6 \mathrm{mg} / \mathrm{ml}$, for $13 \mathrm{~d}$ & Ex vivo aortic ring response & $\begin{array}{l}\text { GTE prevented high-dose angiotensin II decrease in } \\
\text { dilation }\end{array}$ \\
\hline Lim et al. $(2003)^{(47)}$ & $\begin{array}{l}\text { GTE, } 0.3,0.6,1.2 \mathrm{mg} / \mathrm{ml} \\
\text { and EGCG, } 4,12 \mu \mathrm{g} / \mathrm{ml}\end{array}$ & Ex vivo aortic ring response & $\begin{array}{l}\text { Phenylephrine-induced tension dose-dependently inhibited } \\
88-63 \%(P<0.01) \text { with GTE at } 0.6 \text { and } 1.2 \mathrm{mg} / \mathrm{ml} \\
\text { NS for GTE at } 0.3 \mathrm{mg} / \mathrm{ml} \text { and EGCG }\end{array}$ \\
\hline Potenza et al. $(2007)^{(40)}$ & $\begin{array}{l}1-100 \mu \mathrm{M}-\mathrm{EGCG} / 4 \mathrm{~min} \\
200 \mathrm{mg} / \mathrm{kg} \text { per } \mathrm{d} \text { for } 21 \mathrm{~d}\end{array}$ & $\begin{array}{l}\text { Ex vivo aortic ring response } \\
\text { With in vivo supplementation }\end{array}$ & $\begin{array}{l}\text { Dose-dependent vasodilation } \\
\text { Enhanced insulin-mediated vasodilation }(P<0.001)\end{array}$ \\
\hline
\end{tabular}


Lorenz et al. ${ }^{(48)}$, whilst investigating the pathway involved in EGCG and endothelial-dependent vasorelaxation in rat aortic rings, found that EGCG activates endothelial NO synthase (eNOS), a protein which catalyses the production of NO from its precursor L-arginine (Fig. 2). Treatment of bovine endothelial cells with $100 \mu \mathrm{M}$-EGCG caused activation of protein kinase B (Akt) and cAMP-dependent protein kinase A, inhibition of which prevented the increase in eNOS activity. It was demonstrated that Akt was involved in the phosphorylation of eNOS at $\operatorname{Ser}^{1179}$, causing sustained activation whilst protein kinase A was implicated in the rapid activation of eNOS, most probably by phosphorylation. Further experimentation also identified a common upstream phosphorylator in this pathway, phosphatidylinositol-3-OH-kinase, which activates these two kinases. Bovine aortic endothelial cells pretreated with a phosphatidylinositol-3-OH-kinase inhibitor prevented EGCG-induced eNOS activation ${ }^{(48)}$.

Conversely, Alvarez et al. ${ }^{(50)}$ reported EGCG-induced vasorelaxation to be due to its action as a non-selective phosphodiesterase inhibitor, as demonstrated by a failure of EGCG to modify basal generation of cAMP or cGMP but instead instigating a significant reversal of noradrenaline and $\mathrm{KCl}$ inhibition of cAMP and cGMP production in rat aortic smooth muscle cells, albeit at concentrations greater than $25 \mu \mathrm{M}$.

In addition to the rapid impact of EGCG on signalling pathways via an effect on phosphorylation status, there is also evidence to suggest that EGCG may influence the expression of cell signalling genes. Treatment of human umbilical vein endothelial cells (HUVEC) with EGCG treatment $(50 \mu \mathrm{M})$ resulted in specific up- or down-regulation (by more than 1.4-fold) of sixty-five of 12500 genes, many of which are involved in cell signalling ${ }^{(51)}$. Furthermore, chronic EGCG exposure may modulate gene expression by epigenetic changes. Fang et al. ${ }^{(52)}$ have shown EGCG to inhibit DNA methyltransferases in vitro, which has subsequently been related to the proposed cancer-prevention properties of these compounds. However, information regarding the impact of EGCG on epigenetic regulation in relation to the vascular function is currently lacking.

\section{NADPH oxidase inhibitor}

Endothelial NADPH oxidases are involved in pro-inflammatory, proliferative and apoptotic processes through the catalytic production of superoxide free radicals $\left(\mathrm{O}_{2}^{--}\right)$. It has been proposed that catechins may help to regulate NADPH oxidase activity, reducing the production of $\mathrm{O}_{2}^{--}$and thus protecting NO from peroxynitrite formation ${ }^{(53)}$. An increase in $\mathrm{NO}$ bioavailability via reduction in $\mathrm{O}_{2}^{--}$production is a plausible mechanism for the improvement in endothelial function from green tea catechin consumption. Indeed, HUVEC supplementation with mono- $O$-methylated flavanols such as $3^{\prime}$-O-methyl-EC causes a reduction in both the oxidation of NADPH and subsequent $\mathrm{O}_{2}^{--}$release, an inhibitory property not seemingly evident with parent compounds ${ }^{(54)}$. Structural comparison of these mono-methylated flavanols with apocynin, a known NADPH oxidase inhibitor, substantiates the claim of shared function. It is also of interest to note that mono-methylation, demonstrated with (-)-EC methylation at the $3^{\prime}$ and $4^{\prime}$ position, appears to remove the $\mathrm{O}_{2}^{--}$-scavenging ability possessed by the parent compound ${ }^{(54)}$.

\section{Prostacyclin production}

Mizugaki et al. ${ }^{(55)}$ found prostacyclin $\left(\mathrm{PGI}_{2}\right)$ production in cultured bovine endothelial cells to be dose-dependently increased by the addition of EGCG $(25-200 \mu \mathrm{M})$. $\mathrm{PGI}_{2}$ is a known potent vasodilator and thus an increase in $\mathrm{PGI}_{2}$ concentration by EGCG could be a possible mode of action.

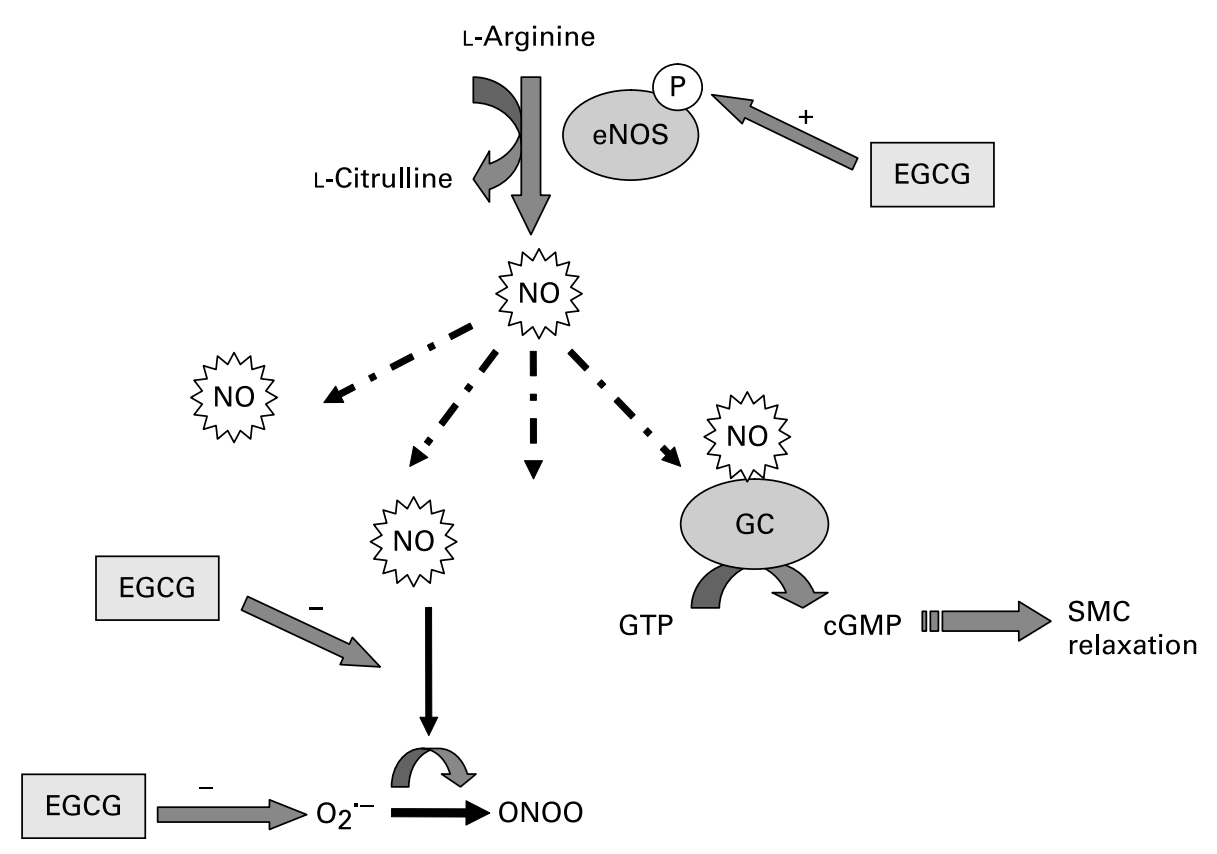

Fig. 2. NO pathway and possible sites of epigallocatechin gallate (EGCG) control in vascular relaxation. eNOS, endothelial NO synthase; $p$, phosphorylated; GC, guanylate cyclase; GTP, guanosine triphosphate; cGMP, cyclic guanosine monophosphate; SMC, smooth muscle cell; ONOO, peroxynitrite. 
This response was much greater for EGCG as compared with other catechins such as EC, catechin and EGC. The addition of gallic acid was also found to increase $\mathrm{PGI}_{2}$ production, indicating the importance of a pyrogallol structure in this pathway. An antioxidative effect similar to that found with $\alpha$-tocopherol may be at play, enhancing $\mathrm{PGI}_{2}$ production. Similarly, a reduction in lipid peroxides, which have an inhibitory effect on $\mathrm{PGI}_{2}$ synthase activity, has also been proposed.

\section{Anti-inflammatory effects}

An EGCG inhibition of endothelial exocytosis and P-selectin cell-surface expression has also been demonstrated by Yamakuchi et al. ${ }^{(56)}$. HUVEC were pretreated with $0-10 \mu \mathrm{M}-\mathrm{EGCG}$ and stimulated with thrombin. EGCG inhibited von Willebrand factor release concentration-dependently and blocked P-selectin translocation. EGCG inhibition of endothelial exocytosis was confirmed by measuring the adherence of labelled leucocytes. The NO inhibitor L-NAME and a PI3K inhibitor reduced the exocytosis-inhibitory effects of EGCG, suggesting a NO-dependent pathway. EC, ECG and EGC had no significant effect ${ }^{(56)}$. Ludwig et al. also found a similar EGCG anti-inflammatory function in that the cytokine induction of VCAM-1 in HUVEC was found to be inhibited by $\mathrm{EGCG}^{(57)}$. The exact pathway has yet to be elucidated but indications point to this activity being the property of gallate group-possessing catechins only. An additional antiinflammatory effect of $(+)$-catechin metabolites has also been found by Koga \& Meydani ${ }^{(14)}$. Pretreatment of human aortic endothelial cells (HAEC) with $7.6 \mu \mathrm{M}-(+)$-catechin metabolites extracted from the plasma of (+)-catechintreated rats significantly inhibited monocyte adhesion, an effect not found with the extract of control plasma or pure $(+)$-catechin ${ }^{(14)}$. The involvement of adhesion molecules in this mechanism was only partly investigated, with the finding of unaltered ICAM, VCAM and E-selectin expression with catechin treatment; further investigation into this inhibition pathway is therefore required.

\section{Asymmetric dimethylarginine reduction}

Asymmetric dimethylarginine (ADMA) has been highlighted as a possible factor in endothelial dysfunction and novel marker of coronary artery disease ${ }^{(58)}$. The endogenous compound is thought to act as an eNOS inhibitor, impairing the production of NO and subsequently decreasing the potential for vasodilation. LDL-induced endothelial dysfunction has also been associated with increased levels of ADMA ${ }^{(59)}$. Interestingly, EGCG has been shown to reduce the level of ADMA along with increasing NO in Sprague-Dawley rats pretreated with LDL. These effects were related to the observed protective effect on endothelial function in aortic rings $^{(60)}$. The EGCG mechanism proposed by the authors is a reduction of the inflammatory cytokine TNF- $\alpha$ and inhibition of lipid peroxidation with consequential enhancement of dimethylaminohydrolase activity, the protein which degrades ADMA. Decreased ADMA may lead to increased $\mathrm{NO}$ and an improvement in endothelial function.

\section{Angiotensin-converting enzyme inhibition}

Angiotensin-converting enzyme (ACE) is an important mediator in blood pressure regulation, converting angiotensin into its activated vasoconstricting form and inactivating the potent vasodilator bradykinin. Green tea and isolated green tea catechins have been found to significantly inhibit the activity of purified and membrane-bound ACE, evaluated by conversion of hippuryl-L-histidyl-L-leucine to hippuric $\operatorname{acid}^{(61)}$. The green tea polyphenol EGC was found to be one of the best inhibitors, with a $50 \%$ inhibitory concentration $\left(\mathrm{IC}_{50}\right)$ in the micromolar range. The authors suggested that hydroxyl groups for hydrogen bonding to ACE and a high molecular mass (dimers and hexamers) were important properties for effective inhibition ${ }^{(61)}$. This mechanism may ultimately contribute to increased vascular health by increasing vasodilation and decreasing blood pressure. It is, however, debatable whether polyphenol dimers and trimers are effectively absorbed into the bloodstream; further studies into this and the mechanism of catechin ACE inhibition are needed.

\section{Evidence of green tea catechins as an antioxidant or pro-oxidant}

Radical-scavenging ability of green tea catechins. There is speculation on whether the effects of the tea catechins are due to their radical-scavenging ability, with oxidative stress and an associated pro-inflammatory response being atherogenic and associated with a loss of vascular function. Protection of LDL from oxidation (reducing the increased accumulation of oxidised LDL in atherosclerotic plaques), metal-chelating abilities or prevention of NO dissolution by free radicals by catechins may help to prevent vascular dysfunction. Nanjo et al. ${ }^{(13)}$ found EGCG to be the most effective tea catechin for free radical (superoxide, hydroxyl and 1,1-diphenyl-2-picrylhydryl (DPPH)) scavenging. The scavenging abilities of the $\alpha$-glucosides were found to vary depending on the position. These investigations led to the conclusion that an ortho-di-hydroxyl group in the $\mathrm{B}$ ring of the tea catechins was an important requirement for radical scavenging and that modification of this group by glucosylation hindered the catechin-scavenging abilities. Although NO is crucial in maintaining endothelial function, the copious production of NO by inducible NO synthase formed by macrophages in the vascular wall and up-regulated by various cytokines induced by cellular inflammation may be detrimental. NO is able to react with superoxide radicals sequentially forming peroxynitrite radicals $\left(\mathrm{ONOO}^{-}\right)$, which, amongst other effects, can proceed to oxidise LDL or up-regulate pro-inflammatory cytokines and adhesion molecules. EGCG inhibition of the excessive NO production and expression of inducible NO synthase has been shown ${ }^{(62)}$ and together with an $\mathrm{ONOO}^{-}$-scavenging effect could be aiding healthy endothelial maintenance. However, convincing evidence for a reduction in ex vivo LDL oxidation in green tea intervention studies has not yet been demonstrated.

Furthermore, uncertainty exists regarding the likely antioxidant benefits of the concentrations of catechins present in tissues and biological fluids relative to other dietary antioxidants, suggesting that the radical-scavenging abilities may 
only partly contribute to the beneficial effects of catechins to the cardiovascular system. The average peak plasma concentration of individual green tea catechins after a single dose of GTE has been reported to be less than $1 \mu \mathrm{mol} / \mathrm{l}^{(63,64)}$ compared with an average plasma concentration of about $55 \mu \mathrm{mol} / 1$ for the antioxidant vitamin $\mathrm{C}^{(65)}$. Although controversial $^{(66-68)}$, an increase in antioxidative ability has, however, been shown after green tea catechin oral administration in selected studies ${ }^{(69-72)}$. In addition, polyphenol metabolites have been indicated as significant contributors to antioxidant capacity, as the calculated concentration of polyphenols needed for the antioxidant capacity value measured after consumption is often higher than that which can be accounted for by solely the parent compounds present in the plasma $^{(73)}$.

Pro-oxidant effect of green tea catechins. Many researchers portray the concept that an alternative mechanism may be responsible. For example, rather than an antioxidant effect, EGCG has been proposed to be operating as a prooxidant. It is well known that dietary antioxidants can also behave as pro-oxidants depending on surrounding conditions. EGCG has been found by Kim et al. to generate $\mathrm{H}_{2} \mathrm{O}_{2}$ in cultured bovine aortic endothelial cells ${ }^{(74)}$. This reactive oxygen species has been shown to phosphorylate Fyn, which in turn has been proposed to activate PI3K, Akt and eNOS, consequentially stimulating the production of NO and mediating the vasodilatory actions observed. Indeed, inhibition of this pathway by $N$-acetylcysteine (reactive oxygen species scavenger), L-NAME, wortmannin (PI3K inhibitor), or pyruvate dehydrogenase kinase-2 (Src family kinase inhibitor) results in an inhibition of EGCG-induced increase in NO production.

The pathway proposed is similar to that employed by insulin, a well-known potent vasodilator, which promotes vasodilation through activation of PI3K, activating pyruvate dehydrogenase kinase isozyme 1 and Akt with sequential eNOS phosphorylation and NO production. Kim et al. ${ }^{(74)}$ have suggested a lack of complete pathway overlap with insulin with regards to its EGCG vasodilatory function. EGCG was shown not to be acting through the insulin receptor for its vasodilatory effects, as unlike with insulin, the receptor was not phosphorylated when cells were treated with EGCG.

Albeit NO-independent, a similar pro-oxidant pathway has also been suggested by $\mathrm{Wu}$ et al. who found transcriptional up-regulation of haeme oxygenase-1, a cytoprotective enzyme, with the addition of $50 \mu \mathrm{M}-\mathrm{EGCG}$ in cultured bovine aortic endothelial cells ${ }^{(75)}$. Activation of both Akt and extracellular signal-regulated kinase (ERK) $1 / 2$ has again been postulated as being involved in this pathway, downstream of PI3K and mitogen-activated protein ERK kinase (MEK) 1/2. Indeed, a protein tyrosine kinase inhibitor, PI3K inhibitors and a MEK1/2 inhibitor were all independently found to attenuate EGCG-induced haeme oxygenase- 1 expression $^{(75)}$. The authors have suggested that a minor increase in reactive oxygen species by EGCG may stimulate this pathway leading to an up-regulation in haeme oxygenase-1 expression. The observed cytoprotective effect of EGCG could thus viably reduce any oxidative stress and thus help to maintain the normal functioning of the vascular endothelium.

\section{Systemic effects of green tea catechins on vascular health} improvement

In addition to endothelial function, green tea catechins have an impact on CVD risk through a number of alternative mechanisms such as cholesterol lowering and increase in insulin sensitivity. Although not directly related to endothelial function, it is recognised that these mechanisms could indirectly improve endothelial function and are therefore included in this section.

Increased insulin activity and sensitivity. In addition to insulin-mimicked vasodilation, green tea has been reported to reduce glucose levels and to possess an 'anti-diabetic' effect in animal ${ }^{(76,77)}$ and cell-culture studies ${ }^{(78)}$. An increase in insulin activity has also been shown with green, black and oolong teas in rat epididymal adipocytes ${ }^{(79)}$. As insulin is a key determinant of eNOS phosphorylation and NO production, insulin sensitivity is imperative for vascular health. The primary active component responsible for the insulin-potentiating effect was found to be EGCG, with ECG, tannins and theaflavins showing a lesser response. An improvement in insulin sensitivity was also found in spontaneously hypertensive rats when supplemented with $200 \mathrm{mg}$ EGCG/kg per d for 3 weeks $^{(40)}$ and Sprague-Dawley rats supplemented with $148 \mathrm{mg}$ green tea catechins/d for $12 \mathrm{~d}^{(80)}$. This has also been replicated more recently in human subjects, in which twelve healthy males given GTE (equivalent to about 3.5 cups green tea) were tested for glucose tolerance. A significant improvement in insulin sensitivity (13\%) and, thus, insulin response to a glucose load was found $(15 \%$ reduction) ${ }^{(81)}$. EGCG supplementation in rat hepatoma cell culture has also shown that EGCG has the ability to interact with the insulin receptor, altering gene expression and increasing specific protein activities, albeit with slower kinetics $^{(78)}$. Thus, EGCG may be able to increase insulin activity and sensitivity, improve vascular health and have applications in metabolic and CVD risk reduction in insulin-resistant individuals ${ }^{(74)}$.

Cholesterol lowering. A hypocholesterolaemic effect has also been shown with green tea catechin administration in animal $^{(82,83)}$ and human studies ${ }^{(84,85)}$. The observed decrease in total cholesterol and LDL-cholesterol levels could be in part responsible for the observed epidemiological reduction in CVD with green tea consumption. It has been proposed that catechins may reduce serum cholesterol levels via reduced cholesterol absorption and increased faecal excretion ${ }^{(86,87)}$. More recently, green tea catechins have also been shown to increase the gene expression of the bile acid biosynthesis enzyme, cholesterol $7 \alpha$-hydroxylase, in HepG2 cells ${ }^{(88)}$. Cholesterol lowering may contribute to the long-term benefits of chronic green tea consumption with regards to vascular health; however, short-term improvements in endothelial function are most likely to be due to an alternative mechanism.

The complete cellular mechanism in which tea catechins exert their beneficial effect is yet to be elucidated. Radicalscavenging activities, anti-inflammatory properties, inhibition or stimulation of specific enzymes and modulation of cell signalling pathways may all contribute to the beneficial endothelial effects from green tea catechin consumption. However, it is worth noting that many of the in vitro and ex vivo studies 
discussed use non-physiological concentrations of tea catechins, frequently at levels 100 -fold greater than plasma levels; therefore results should be interpreted with caution. Further work is needed, specifically on physiological concentrations of green tea catechins and on the metabolites of green tea catechins found in the plasma after digestion and absorption.

\section{Inter- and intra-study variability in epidemiological and human intervention trials}

There is a considerable amount of contradictory evidence in the literature regarding the significance of the effect of green tea on vascular function and cardiovascular health in humans and the 'size' of the effect.

\section{Inter-study variability}

The heterogeneity found in epidemiological studies may be in part attributed to inconsistent or incomplete correction of statistical models for a myriad of physiological and behavioural confounding factors, and inaccurate assessment of tea and catechin intakes. For example, in the UK, smoking is positively associated with tea intake and smoking is a known potent vasoconstrictor, thus any benefits of tea catechins in the prevention of CVD may be negated. In addition, a Japanese study of 8552 individuals found an increasing percentage of male smokers among those with the highest green tea consumption. This observation was speculated to be counteracting the beneficial effects of green tea as an increased cancer incidence was found amongst males in this green tea group whilst a significant protective effect of high green tea consumption was found in females ${ }^{(30)}$. It is also viable that glucuronidation of catechins is modified by lifestyle factors such as diet, smoking, oral contraceptives and alcohol use via competitive inhibition of the glucuronidation enzymes. For example, it has been shown that diet can affect the levels of UGT. Green tea administered to rats for 4 weeks enhanced UGT activity by $100 \%{ }^{(89)}$. Measurement of these confounding factors is often problematical in epidemiological studies as a result of unavoidable respondent bias and changes in lifestyle. Thus, masking effects of residual confounders remains an ongoing issue in such studies, particularly those of lower statistical power.

The basis for the inter-study variability in the responses evident in intervention studies may be attributable to a number of factors. Differences in the type of product given to the volunteers, the dose of catechins administered (which is often dependent on the catechin content of the particular green tea or GTE used in the study ${ }^{(90)}$ ) and its instruction for use will undoubtedly influence the study outcomes. For instance, green tea preparation could conceivably affect the extent of the vascular response by altering the nature and amount of green tea catechins available in the test beverage. Green et al. found in vitro digestive recovery of green tea catechins to be increased when formulated with additives such as milk, citrus juice and ascorbic acid ${ }^{(91)}$. The use of individual catechins in intervention trials as opposed to mixed catechins could also influence the study outcome. Mixed catechins are thought to act as competitive inhibitors of methylation, sulfation and glucuronidation, unlike pure compounds which have no drug-drug interactions. This results in a lowered plasma maximum concentration and area under the concentration curve with pure compounds such as EGCG as compared with green tea, mixed green tea catechins or $\mathrm{GTE}^{(10)}$.

Food intake has been shown to affect tea catechin oral bioavailability. For example, Chow et al. found a 3.5-fold increase in plasma free EGCG when Polyphenon E (decaffeinated green tea catechin mixture) was taken in the fasting condition compared with when taken with food ${ }^{(92)}$. A range of factors may be responsible for this reduction in bioavailability. An altered stomach $\mathrm{pH}$ as a result of feeding could result in catechin instability. Indeed, catechins are known to be more unstable in neutral and alkaline conditions resulting in degradation, oxidation and/or polymerisation of free catechins ${ }^{(10)}$. A depletion in metabolic reaction precursors during an acute fast, such as those needed for glucuronidation and sulfation reactions, could also occur resulting in a decrease in pre-systemic metabolism and a sequential increase in free catechins in the plasma ${ }^{(92)}$. A decrease in catechin and food complexation or interactions may also account for the increase in catechin bioavailability when fasted. Thus, supplementation of green tea catechins with or after a meal may result in poor bioavailability and a subsequent reduction in vascular response. When consumed with a meal, the type of food consumed may also have an effect. For example, the absorption of cocoa flavanols has been shown to increase when consumed with carbohydrates such as bread ${ }^{(93)}$. Therefore inter-study differences in the timing of EGCG consumption relative to habitual meal intakes is likely to have a large impact on the bioavailability of catechins and therefore their physiological effects.

Other aspects of study methodology can also affect findings. The range of methods and protocols available for non-invasive vascular function research does not allow for easy study comparison. Furthermore, there are limited published data comparing the methods, in particular the newer non-invasive techniques, with the best-established yet invasive venousocclusion plethysmography technique. Thus, standardisation of measurement techniques is firstly required, followed by further research into the extent of correlation between techniques before studies utilising different vascular function measurements can be clustered.

\section{Intra-study variability}

A large inter-individual variation in bioavailability of green tea catechins can also be seen in the majority of intervention and pharmacokinetic studies. A number of aforementioned factors may also have an intra-study effect. Lifestyle factors, such as habitual diet, smoking status, alcohol intake and individual characteristics such as BMI and age will give rise to individual differences in response as a result of alterations in catechin bioavailability. Indeed, lifestyle and environmental influences are most likely to be responsible for the intraindividual variations found in acute pharmacokinetic studies where repeated experiments were performed. For example, a pharmacokinetic study conducted by Lee et al. found participant EGCG maximum concentration and area under the concentration curve intra-individual $\mathrm{CV}$ values to be as high as 81 and $68 \%$, respectively, when the experiment was 
repeated on the same group of participants on three separate occasions ${ }^{(5)}$. This variation indicates the influence of environmental factors.

Individual differences in intestinal, microbial and hepatic metabolism may also in part provide an explanation for intra-study variation (and may also be responsible for a large component of inter-study variability, in particular when comparing studies conducted in groups geographically and ethnically divergent). Genetic polymorphisms in the enzymes governing the metabolic transformation of tea catechins such as catechol- $O$-methyltransferase, UGT and sulfotransferases may be partly responsible for the observed variability in flavonoid bioavailability. For example, polymorphisms have been found in the catechol- $O$-methyltransferase gene, resulting in an enzyme with a 3- to 4-fold difference in metabolic ability ${ }^{(94)}$. The glucuronidation of many drugs and steroid hormones have also been shown to be affected by UGT polymorphisms ${ }^{(95)}$, with a recent report of a 25 -fold inter-individual difference in the rate of UGT1A6 glucuronidation ${ }^{(96)}$. The effect of these polymorphisms on catechin bioavailability is yet to be investigated. The genetic variation of receptors, transporters and regulatory proteins could also have an impact on catechin absorption. For example, the multidrug resistance-associated protein 2 (MRP2), an ATP-binding cassette (ABC) intestinal membrane-bound transporter involved in the efflux of catechins back into the lumen, has been found to have several common single nucleotide polymorphisms, some of which have been suggested to have altered expression and function $^{(97)}$.

Differences between individuals with respect to gastrointestinal function may also influence the metabolism and absorption of the catechins. Green tea catechins are relatively poorly absorbed, with bioavailability dependent on many factors within the small intestine such as lipophilicity, solubility, $\mathrm{pH}$ of the lumen, gastrointestinal transit time, mucosal mass and membrane permeability. The range of factors involved in this initial stage could greatly influence the rate and extent of catechin absorption. Indeed, when EGCG stability is increased, absorption is enhanced ${ }^{(98)}$.

The importance of microbial degradation of tea catechins has been mentioned previously. Microbial populations in the gut are highly variable and are specific to the food components that they metabolise. The metabolites produced after microbial degradation are also subject to variation depending on the resident microbial species. As a consequence, inter-individual differences in microflora profile can have a large impact on bioavailability and biotransformation of tea catechins. For example, the isoflavone daidzein is degraded to equol, a non-steroidal oestrogen, by colonic bacteria in only $30-40 \%$ of individuals. Consequentially, equol producers are thought to derive a greater benefit from isoflavones compared with non-producers especially with regards to cardiovascular, bone and menopausal health ${ }^{(99)}$. The levels of the microbial ring-fission metabolites

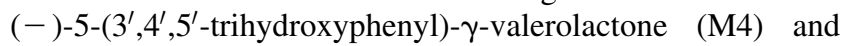
(-)-5-(3',4'-dihydroxyphenyl)- $\gamma$-valerolactone (M6) in plasma and urine after green tea catechin administration have also been shown to differ greatly between individuals ${ }^{(7)}$, which is most likely to be directly related to the variation in gut microbial populations.
Although it is recognised that a whole array of behavioural, physiological and genetic factors have an impact on the metabolism and responsiveness to dietary catechins, such information is often unavailable to add as a covariate to statistical models. However, consideration of these factors when designing studies or evaluating results is advisable.

\section{Conclusion}

There is accumulating evidence from human, animal and cellculture models to indicate that green tea catechins have a positive impact on endothelial and overall vascular functions, with a number of plausible molecular mechanisms proposed. However, these findings are presently inconclusive and not entirely consistent, signifying the need for further research. There is also a concern with the physiological relevance of some in vitro findings which use green tea catechin concentrations far higher than what is achievable through the diet. Additional studies are also required to determine the importance of the metabolised compounds as opposed to the pure compounds, as tissues in humans are often exposed to the metabolites rather than the parent compounds present in food. Wide intra- and inter-individual variation in bioavailability has also been recognised. A greater understanding of this variation is needed to allow specific claims to be made regarding likely dose-response relationships in population subgroups.

\section{Acknowledgements}

R. J. M. drafted the manuscript, with all authors deciding on the review content and contributing to the final version. A. M. M. and K. G. J. have a number of ongoing collaborative projects with Unilever Discover (Colworth Science Park, Sharnbrook, Bedford, Beds, UK). Unilever Discover sponsor R. J. M.'s PhD studies.

\section{References}

1. Higdon JV \& Frei B (2003) Tea catechins and polyphenols: health effects, metabolism, and antioxidant functions. Crit Rev Food Sci Nutr 43, 89-143.

2. Pharn-Huy LAN, He H \& Phamhuy C (2008) Green tea and health: an overview. J Food Agric Environ 6, 6-13.

3. Miyazawa T (2000) Absorption, metabolism and antioxidative effects of tea catechin in humans. Biofactors 13, 55-59.

4. Manach C, Williamson G, Morand C, et al. (2005) Bioavailability and bioefficacy of polyphenols in humans. I. Review of 97 bioavailability studies. Am J Clin Nutr 81, 230S-242S

5. Lee MJ, Maliakal P, Chen LS, et al. (2002) Pharmacokinetics of tea catechins after ingestion of green tea and (-)-epigallocatechin-3-gallate by humans: formation of different metabolites and individual variability. Cancer Epidemiol Biomarkers Prev 11, 1025-1032.

6. Warden BA, Smith LS, Beecher GR, et al. (2001) Catechins are bioavailable in men and women drinking black tea throughout the day. J Nutr 131, 1731-1737.

7. Li C, Lee MJ, Sheng SQ, et al. (2000) Structural identification of two metabolites of catechins and their kinetics in human urine and blood after tea ingestion. Chem Res Toxicol 13, $177-184$. 
8. Lu H, Meng XF \& Yang CS (2003) Enzymology of methylation of tea catechins and inhibition of catechol- $O$-methyltransferase by (-)-epigallocatechin gallate. Drug Metab Dispos 31, $572-579$.

9. Kuhnle G, Spencer JP, Schroeter H, et al. (2000) Epicatechin and catechin are $O$-methylated and glucuronidated in the small intestine. Biochem Biophys Res Commun 277, 507-512.

10. Feng WY (2006) Metabolism of green tea catechins: an overview. Curr Drug Metab 7, 755-809.

11. Lu H, Meng XF, Li C, et al. (2003) Glucuronides of tea catechins: enzymology of biosynthesis and biological activities. Drug Metab Dispos 31, 452-461.

12. Lambert JD, Rice JE, Hong J, et al. (2005) Synthesis and biological activity of the tea catechin metabolites, M4 and M6 and their methoxy-derivatives. Bioorg Med Chem Lett 15, 873-876.

13. Nanjo F, Mori M, Goto K, et al. (1999) Radical scavenging activity of tea catechins and their related compounds. Biosci Biotechnol Biochem 63, 1621-1623.

14. Koga T \& Meydani M (2001) Effect of plasma metabolites of $(+)$-catechin and quercetin on monocyte adhesion to human aortic endothelial cells. Am J Clin Nutr 73, 941-948.

15. Cybulsky MI \& Gimbrone MA Jr (1991) Endothelial expression of a mononuclear leukocyte adhesion molecule during atherogenesis. Science 251, 788-791.

16. Rohde LE, Lee RT, Rivero J, et al. (1998) Circulating cell adhesion molecules are correlated with ultrasound-based assessment of carotid atherosclerosis. Arterioscler Thromb Vasc Biol 18, 1765-1770.

17. Dong ZM, Chapman SM, Brown AA, et al. (1998) The combined role of $\mathrm{P}$ - and E-selectins in atherosclerosis. J Clin Invest 102, 145-152.

18. Collins RG, Velji R, Guevara NV, et al. (2000) P-selectin or intercellular adhesion molecule (ICAM)-1 deficiency substantially protects against atherosclerosis in apolipoprotein E-deficient mice. J Exp Med 191, 189-194.

19. Schachinger V, Britten MB \& Zeiher AM (2000) Prognostic impact of coronary vasodilator dysfunction on adverse longterm outcome of coronary heart disease. Circulation 101, $1899-1906$

20. Anderson TJ, Uehata A, Gerhard MD, et al. (1995) Close relation of endothelial function in the human coronary and peripheral circulations. J Am Coll Cardiol 26, 1235-1241.

21. Heitzer T, Schlinzig T, Krohn K, et al. (2001) Endothelial dysfunction, oxidative stress, and risk of cardiovascular events in patients with coronary artery disease. Circulation 104, 2673-2678.

22. Halcox JPJ, Schenke WH, Zalos G, et al. (2002) Prognostic value of coronary vascular endothelial dysfunction. Circulation 106, 653-658.

23. Landmesser U, Hornig B \& Drexler H (2004) Endothelial function - a critical determinant in atherosclerosis? Circulation 109, 27-33

24. Chan NN, Colhoun HM \& Vallance P (2001) Cardiovascular risk factors as determinants of endothelium-dependent and endothelium-independent vascular reactivity in the general population. J Am Coll Cardiol 38, 1814-1820.

25. Imai K \& Nakachi K (1995) Cross sectional study of effects of drinking green tea on cardiovascular and liver diseases. $B M J$ 310, 693-696.

26. Sano J, Inami S, Seimiya K, et al. (2004) Effects of green tea intake on the development of coronary artery disease. Circ $J$ 68, 665-670.

27. Sasazuki S, Kodama H, Yoshimasu K, et al. (2000) Relation between green tea consumption and the severity of coronary atherosclerosis among Japanese men and women. Ann Epidemiol 10, 401-408.
28. Hirano R, Momiyama Y, Takahashi R, et al. (2002) Comparison of green tea intake in Japanese patients with and without angiographic coronary artery disease. Am J Cardiol 90, $1150-1153$.

29. Kuriyama S, Shimazu T, Ohmori K, et al. (2006) Green tea consumption and mortality due to cardiovascular disease, cancer, and all causes in Japan: the Ohsaki study. JAMA 296, $1255-1265$.

30. Nakachi K, Matsuyama S, Miyake S, et al. (2000) Preventive effects of drinking green tea on cancer and cardiovascular disease: epidemiological evidence for multiple targeting prevention. Biofactors 13, 49-54.

31. Widlansky ME, Hamburg NM, Anter E, et al. (2007) Acute EGCG supplementation reverses endothelial dysfunction in patients with coronary artery disease. $J$ Am Coll Nutr 26, 95-102.

32. Ryu OH, Lee J, Lee KW, et al. (2006) Effects of green tea consumption on inflammation, insulin resistance and pulse wave velocity in type 2 diabetes patients. Diabetes Res Clin Pract 71, 356-358.

33. Kim W, Jeong MH, Cho SH, et al. (2006) Effect of green tea consumption on endothelial function and circulating endothelial progenitor cells in chronic smokers. Circ J 70, $1052-1057$.

34. Tinahones Madueño FJ, Rubio MA, Garrido Sánchez L, et al. (2007) Green tea reduces LDL oxidability and improves vascular function. Atheroscler Suppl 8, 166.

35. Lee W, Min WK, Chun S, et al. (2005) Long-term effects of green tea ingestion on atherosclerotic biological markers in smokers. Clin Biochem 38, 84-87.

36. Jochmann N, Lorenz M, Krosigk AV, et al. (2008) The efficacy of black tea in ameliorating endothelial function is equivalent to that of green tea. Br J Nutr 99, $863-868$.

37. Nagaya N, Yamamoto H, Uematsu M, et al. (2004) Green tea reverses endothelial dysfunction in healthy smokers. Heart $\mathbf{9 0}$, $1485-1486$.

38. Alexopoulos N, Vlachopoulos C, Aznaouridis K, et al. (2008) The acute effect of green tea consumption on endothelial function in healthy individuals. Eur J Cardiovasc Prev Rehabil 15, 300-305

39. Antonello M, Montemurro D, Bolognesi M, et al. (2007) Prevention of hypertension, cardiovascular damage and endothelial dysfunction with green tea extracts. Am J Hypertens 20, $1321-1328$.

40. Potenza MA, Marasciulo FL, Tarquinio M, et al. (2007) EGCG, a green tea polyphenol, improves endothelial function and insulin sensitivity, reduces blood pressure, and protects against myocardial I/R injury in SHR. Am J Physiol Endocrinol Metab 292, E1378-E1387.

41. Chyu KY, Babbidge SM, Zhao X, et al. (2004) Differential effects of green tea-derived catechin on developing versus established atherosclerosis in apolipoprotein E-null mice. Circulation 109, 2448-2453.

42. Miura Y, Chiba T, Tomita I, et al. (2001) Tea catechins prevent the development of atherosclerosis in apoprotein E-deficient mice. J Nutr 131, 27-32.

43. Song L, Koo MWL, Lau CP, et al. (2008) Effects of green tea on lipids, blood pressure and vasorelaxation in rats with hypercholesterolaemia-induced hypertension. Int $J$ Cardiol 125, Suppl. 1, S64.

44. Sanae F, Miyaichi Y, Kizu H, et al. (2002) Effects of catechins on vascular tone in rat thoracic aorta with endothelium. Life Sci 71, 2553-2562.

45. Ajay M, Gilani AU \& Mustafa MR (2003) Effects of flavonoids on vascular smooth muscle of the isolated rat thoracic aorta. Life Sci 74, 603-612. 
46. Fitzpatrick DF, Hirschfield SL, Ricci T, et al. (1995) Endothelium-dependent vasorelaxation caused by various plant extracts. J Cardiovasc Pharmacol 26, 90-95.

47. Lim DY, Lee ES, Park HG, et al. (2003) Comparison of green tea extract and epigallocatechin gallate on blood pressure and contractile responses of vascular smooth muscle of rats. Arch Pharm Res 26, 214-223.

48. Lorenz M, Wessler S, Follmann E, et al. (2004) A constituent of green tea, epigallocatechin-3-gallate, activates endothelial nitric oxide synthase by a phosphatidylinositol-3-OH-kinase-, cAMP-dependent protein kinase-, and Akt-dependent pathway and leads to endothelial-dependent vasorelaxation. J Biol Chem 279, 6190-6195.

49. Shen JZ, Zheng XF, Wei EQ, et al. (2003) Green tea catechins evoke a phasic contraction in rat aorta via $\mathrm{H}_{2} \mathrm{O}_{2}$-mediated multiple-signalling pathways. Clin Exp Pharmacol Physiol 30, $88-95$.

50. Alvarez E, Campos-Toimil M, Justiniano-Basaran $\mathrm{H}$, et al. (2006) Study of the mechanisms involved in the vasorelaxation induced by (-)-epigallocatechin-3-gallate in rat aorta. $\mathrm{Br} \mathrm{J}$ Pharmacol 147, 269-280.

51. Pfeffer U, Ferrari N, Dell'Eva R, et al. (2005) Molecular mechanisms of action of angiopreventive anti-oxidants on endothelial cells: microarray gene expression analyses. Mutat Res 591, 198-211.

52. Fang M, Chen D \& Yang CS (2007) Dietary polyphenols may affect DNA methylation. J Nutr 137, Suppl. 1, 223S-228S.

53. Schewe T, Steffen Y \& Sies H (2008) How do dietary flavanols improve vascular function? A position paper. Arch Biochem Biophys 476, 102-106.

54. Steffen Y, Gruber C, Schewe T, et al. (2008) Mono- $O$-methylated flavanols and other flavonoids as inhibitors of endothelial NADPH oxidase. Arch Biochem Biophys 469, 209-219.

55. Mizugaki M, Ishizawa F, Yamazaki T, et al. (2000) Epigallocatechin gallate increase the prostacyclin production of bovine aortic endothelial cells. Prostaglandins Other Lipid Mediat 62, 157-164.

56. Yamakuchi M, Bao C, Ferlito M, et al. (2008) Epigallocatechin gallate inhibits endothelial exocytosis. Biol Chem 389, 935-941.

57. Ludwig A, Lorenz M, Grimbo N, et al. (2004) The tea flavonoid epigallocatechin-3-gallate reduces cytokine-induced VCAM-1 expression and monocyte adhesion to endothelial cells. Biochem Biophys Res Commun 316, 659-665.

58. Boger RH (2003) Asymmetric dimethylarginine (ADMA) modulates endothelial function - therapeutic implications. Vasc Med 8, 149-151.

59. Jiang JL, Jiang DJ, Tang YH, et al. (2004) Effect of simvastatin on endothelium-dependent vaso-relaxation and endogenous nitric oxide synthase inhibitor. Acta Pharmacol Sin 25, 893-901.

60. Tang WJ, Hu CP, Chen MF, et al. (2006) Epigallocatechin gallate preserves endothelial function by reducing the endogenous nitric oxide synthase inhibitor level. Can J Physiol Pharmacol 84, 163-171.

61. Actis-Goretta L, Ottaviani JI \& Fraga CG (2006) Inhibition of angiotensin converting enzyme activity by flavanol-rich foods. J Agric Food Chem 54, 229-234.

62. Paquay JB, Haenen GR, Stender G, et al. (2000) Protection against nitric oxide toxicity by tea. J Agric Food Chem 48, 5768-5772.

63. Chow HHS, Cai Y, Alberts DS, et al. (2001) Phase I pharmacokinetic study of tea polyphenols following single-dose administration of epigallocatechin gallate and polyphenon E. Cancer Epidemiol Biomarkers Prev 10, 53-58.

64. Yang CS, Chen LS, Lee MJ, et al. (1998) Blood and urine levels of tea catechins after ingestion of different amounts of green tea by human volunteers. Cancer Epidemiol Biomarkers Prev 7, 351-354.

65. Ruston D, Hoare J, Henderson L, et al. (2004) National Diet and Nutrition Survey: Adults Aged 19 to 64 Years. Volume 4: Nutritional Status (Anthropometry and Blood Analytes), Blood Pressure and Physical Activity. London: FSA.

66. Duffy SJ, Keaney JF Jr, Holbrook M, et al. (2001) Short- and long-term black tea consumption reverses endothelial dysfunction in patients with coronary artery disease. Circulation 104, $151-156$.

67. Hodgson JM, Puddey IB, Croft KD, et al. (2000) Acute effects of ingestion of black and green tea on lipoprotein oxidation. Am J Clin Nutr 71, 1103-1107.

68. Lotito SB \& Frei B (2006) Consumption of flavonoid-rich foods and increased plasma antioxidant capacity in humans: cause, consequence, or epiphenomenon? Free Radic Biol Med 41, 1727-1746.

69. Da Silva EL, Piskula M \& Terao J (1998) Enhancement of antioxidative ability of rat plasma by oral administration of (-)-epicatechin. Free Radic Biol Med 24, 1209-1216.

70. Benzie IF, Szeto YT, Strain JJ, et al. (1999) Consumption of green tea causes rapid increase in plasma antioxidant power in humans. Nutr Cancer 34, 83-87.

71. Leenen R, Roodenburg AJ, Tijburg LB, et al. (2000) A single dose of tea with or without milk increases plasma antioxidant activity in humans. Eur J Clin Nutr 54, 87-92.

72. Erba D, Riso P, Bordoni A, et al. (2005) Effectiveness of moderate green tea consumption on antioxidative status and plasma lipid profile in humans. J Nutr Biochem 16, 144-149.

73. Scalbert A \& Williamson G (2000) Dietary intake and bioavailability of polyphenols. J Nutr 130, Suppl. 8S, 2073S-2085S.

74. Kim JA, Formoso G, Li Y, et al. (2007) Epigallocatechin gallate, a green tea polyphenol, mediates NO-dependent vasodilation using signaling pathways in vascular endothelium requiring reactive oxygen species and Fyn. J Biol Chem 282, 13736-13745.

75. Wu CC, Hsu MC, Hsieh CW, et al. (2006) Upregulation of heme oxygenase- 1 by epigallocatechin-3-gallate via the phosphatidylinositol 3-kinase/Akt and ERK pathways. Life Sci 78, 2889-2897.

76. Shenoy C (2000) Hypoglycemic activity of bio-tea in mice. Indian J Exp Biol 38, 278-279.

77. Renno WM, Abdeen S, Alkhalaf M, et al. (2008) Effect of green tea on kidney tubules of diabetic rats. Br J Nutr 100, 652-659.

78. Waltner-Law ME, Wang XL, Law BK, et al. (2002) Epigallocatechin gallate, a constituent of green tea, represses hepatic glucose production. J Biol Chem 277, 34933-34940.

79. Anderson RA \& Polansky MM (2002) Tea enhances insulin activity. J Agric Food Chem 50, 7182-7186.

80. Wu LY, Juan CC, Ho LT, et al. (2004) Effect of green tea supplementation on insulin sensitivity in Sprague-Dawley rats. J Agric Food Chem 52, 643-648.

81. Venables MC, Hulston CJ, Cox HR, et al. (2008) Green tea extract ingestion, fat oxidation, and glucose tolerance in healthy humans. Am J Clin Nutr 87, 778-784.

82. Ramesh E, Elanchezhian R, Sakthivel M, et al. (2008) Epigallocatechin gallate improves serum lipid profile and erythrocyte and cardiac tissue antioxidant parameters in Wistar rats fed an atherogenic diet. Fundam Clin Pharmacol 22, 275-284.

83. Yang TT \& Koo MW (1997) Hypocholesterolemic effects of Chinese tea. Pharmacol Res 35, 505-512.

84. Kono S, Shinchi K, Wakabayashi K, et al. (1996) Relation of green tea consumption to serum lipids and lipoproteins in Japanese men. J Epidemiol 6, 128-133. 
85. Tokunaga S, White IR, Frost C, et al. (2002) Green tea consumption and serum lipids and lipoproteins in a population of healthy workers in Japan. Ann Epidemiol 12, $157-165$.

86. Koo SI \& Noh SK (2007) Green tea as inhibitor of the intestinal absorption of lipids: potential mechanism for its lipidlowering effect. J Nutr Biochem 18, 179-183.

87. Yang TTC \& Koo MWL (2000) Chinese green tea lowers cholesterol level through an increase in fecal lipid excretion. Life Sci 66, 411-423.

88. Lee MS, Park JY, Freake H, et al. (2008) Green tea catechin enhances cholesterol $7 \alpha$-hydroxylase gene expression in HepG2 cells. Br J Nutr 99, 1182-1185.

89. Bu-Abbas A, Clifford MN, Ioannides C, et al. (1995) Stimulation of rat hepatic UDP-glucuronosyl transferase activity following treatment with green tea. Food Chem Toxicol 33, $27-30$.

90. Khokhar S \& Magnusdottir SG (2002) Total phenol, catechin, and caffeine contents of teas commonly consumed in the United Kingdom. J Agric Food Chem 50, 565-570.

91. Green RJ, Murphy AS, Schulz B, et al. (2007) Common tea formulations modulate in vitro digestive recovery of green tea catechins. Mol Nutr Food Res 51, 1152-1162.

92. Chow HHS, Hakim IA, Vining DR, et al. (2005) Effects of dosing condition on the oral bioavailability of green tea catechins after single-dose administration of Polyphenon E in healthy individuals. Clin Cancer Res 11, 4627-4633.
93. Schramm DD, Karim M, Schrader HR, et al. (2003) Food effects on the absorption and pharmacokinetics of cocoa flavanols. Life Sci 73, 857-869.

94. Dawling S, Roodi N, Mernaugh RL, et al. (2001) Catechol-Omethyltransferase (COMT)-mediated metabolism of catechol estrogens: comparison of wild-type and variant COMT isoforms. Cancer Res 61, 6716-6722.

95. Lampe JW (2007) Diet, genetic polymorphisms, detoxification, and health risks. Altern Ther Health Med 13, S108-S111.

96. Nagar S, Zalatoris JJ \& Blanchard RL (2004) Human UGT1A6 pharmacogenetics: identification of a novel SNP, characterization of allele frequencies and functional analysis of recombinant allozymes in human liver tissue and in cultured cells. Pharmacogenetics 14, 487-499.

97. Sai K, Saito Y, Itoda M, et al. (2008) Genetic variations and haplotypes of ABCC2 encoding MRP2 in a Japanese population. Drug Metab Pharmacokinet 23, 139-147.

98. Gawande S, Kale A \& Kotwal S (2008) Effect of nutrient mixture and black grapes on the pharmacokinetics of orally administered (-)epigallocatechin-3-gallate from green tea extract: a human study. Phytother Res 22, 802-808.

99. Setchell KD, Brown NM \& Lydeking-Olsen E (2002) The clinical importance of the metabolite equol - a clue to the effectiveness of soy and its isoflavones. J Nutr 132, 3577-3584.

100. Del Rio D, Stewart AJ, Mullen W, et al. (2004) HPLC-MS ${ }^{\mathrm{n}}$ analysis of phenolic compounds and purine alkaloids in green and black tea. J Agric Food Chem 52, 2807-2815. 